\title{
Der feine Unterschied - Genderspezifische Aspekte psychosozialer Risikofaktoren und Interventionen bei Patienten mit koronarer Herzkrankheit
}

\author{
Alexandra Boese $\cdot$ Christoph Herrmann-Lingen
}

Online publiziert: 19. März 2013

(C) Springer-Verlag Berlin Heidelberg 2013

Zusammenfassung In den vergangenen 20 Jahren hat die Sensibilität für genderspezifische Aspekte in der Erforschung von psychosozialen Risikofaktoren und Interventionen bei KHK deutlich zugenommen. Die vorliegende Übersicht zeigt, dass die geschlechtsspezifischen Unterschiede bei den psychosozialen Risikofaktoren gering, aber bedeutsam sind. Psychosoziale Interventionen sind geeignet, Belastungen zu lindern. Bisher ist jedoch nicht eindeutig nachgewiesen, ob sie auch die KHK selbst und die Mortalität beeinflussen.

Schlüsselwörter Gender · Koronare Herzkrankheit ·

Psychosozial · Risikofaktoren · Interventionen

The subtle distinction: specific aspects of psychosocial risk factors and interventions in men and women with coronary heart disease

\begin{abstract}
During the past 20 years research on genderspecific aspects in psychosocial risk factors and interventions for $\mathrm{CHD}$ has widely increased. In a literature update we show that gender-specific differences in psychosocial risk factors are small but relevant. Psychosocial interventions reduce distress but it is still under discussion if they improve the course of CHD itself and related mortality.
\end{abstract}

Keywords Gender · Coronary heart disease ·

Psychosocial $\cdot$ Risk factors $\cdot$ Interventions

Dipl.-Psych. A. Boese $(\bowtie) \cdot$ Prof. Dr. C. Herrmann-Lingen Abteilung für Psychosomatische Medizin und Psychotherapie, Universitätsmedizin Göttingen, Von-Siebold-Str. 5,

37075 Göttingen, Deutschland

E-Mail: alexandra.boese@med.uni-goettingen.de

\section{Hintergrund}

Bei Entstehung und Verlauf einer koronaren Herzerkrankung (KHK) spielen psychosoziale Belastungen eine wichtige, unabhängige Rolle. So machen psychosoziale Risikofaktoren (siehe Box 1) etwa 30\% des Gesamtrisikos für einen Myokardinfarkt aus [1]. Die Beachtung psychosozialer Risikofaktoren ist daher für die Prävention und Behandlung der KHK bedeutsam.

Für Männer und Frauen ergeben sich unterschiedliche psychosoziale Erfahrungen und daraus resultierende Belastungen. Während die Forschung zu psychosozialen Risikofaktoren und Interventionen bei KHK in der Vergangenheit hauptsächlich Männer berücksichtigte, werden heute Studien auch mit Frauen durchgeführt. Dies bietet das Potenzial, Risikofaktoren geschlechtsspezifisch zu differenzieren und Interventionen wirksamer zu gestalten.

Box 1: Mit erhöhtem KHK-Risiko assoziierte psychosoziale Merkmale

- Externe Stressoren:

- Niedriger sozioökonomischer Status

- Mangelnde soziale Unterstützung

- Familiäre Konflikte

- Chronische Arbeitsbelastung

- Doppelbelastung durch Beruf und Familie (spezifisch bei Frauen)

- Interne Stressoren:

- Depressivität

- Pathologische Angst

- Feindseligkeit

- Unterdrückter Ärger (spezifisch bei Frauen) 


\section{Genderspezifische Aspekte psychosozialer Risikofaktoren}

Psychosoziale KHK-Risikofaktoren lassen sich in zwei Kategorien einteilen, nämlich (externe) Stressoren im Sinne anhaltender Belastungen auf der einen und (interne) emotionale Belastungen auf der anderen Seite.

Erhöhtes kardiales Risiko durch anhaltende Belastungen bzw. Stress

Anhaltende Stressbelastungen sind ein anerkannter psychosozialer KHK-Risikofaktor [2]. Für beide Geschlechter bedeutet mangelnde soziale Unterstützung ein erhöhtes Risiko für die Entwicklung einer KHK und einen ungünstigeren Krankheitsverlauf [3]. Während der Ehestand für Männer anders als für Frauen ein insgesamt protektiver Faktor zu sein scheint, erhöhen Belastungen in der Ehe das Risiko, einen ersten Myokardinfarkt zu erleiden [4-6].

Frauen haben nach einem kardialen Ereignis bei Belastungen in der Ehe ein dreifach erhöhtes Risiko für ein erneutes kardiales Ereignis im Vergleich zu unbelasteten Frauen [7]. Das Risiko erhöht sich weiter bei gleichzeitig hoher Arbeitsbelastung (,high strain jobs" nach Karasek [8], d. h. hohe Anforderungen bei verhältnismäßig geringem Autonomieerleben) [9].

Hohe Arbeitsbelastung und Gratifikationskrisen im Beruf beinhalten für Männer ein bedeutsames Risiko für die Entstehung einer KHK oder eines ersten Herzinfarkts [10-13]. Für die KHK-Inzidenz scheint beruflicher Stress bei Frauen eine ebenso bedeutsame Rolle zu spielen wie bei Männern. Allerdings gibt es Hinweise dafür, dass bei Frauen eine Berufstätigkeit, die hohe Anforderungen stellt und hohe Autonomie mit sich bringt (,active jobs“ im ,job strain“; Modell nach Karasek [8]), ein höheres Risiko bedeutet [14, 15], während Männer bei hohen Anforderungen und geringer Autonomie einem höheren Risiko ausgesetzt sind. Zudem könnte bei Frauen eine subjektiv geringe berufliche Belastung gegenüber dem Fehlen einer Berufstätigkeit günstige Effekte haben [16].

Bei Frauen, die bereits kardial erkrankt sind, scheint die Arbeitsbelastung allein nicht im Zusammenhang mit weiteren kardialen Ereignissen zu stehen [17]. Durch die Doppelbelastung durch Arbeitsbelastung und Eheprobleme wird ein erneutes kardiales Ereignis jedoch deutlich wahrscheinlicher als ohne diese Belastungen [9]. Allerdings gibt es Hinweise, dass nach einem Herzinfarkt ein chronisch erhöhter Berufsstress für beide Geschlechter im mehrjährigen Verlauf eine erhöhte kardiale Ereignisrate vorhersagt [18].
Erhöhtes kardiales Risiko durch emotionale Belastungen

Eine depressive Erkrankung bedeutet für initial herzgesunde Personen ein nahezu verdoppeltes Risiko, an einer koronaren Herzerkrankung zu erkranken und stellt somit einen wichtigen unabhängigen psychosozialen Risikofaktor dar [19-22]. Frauen mit KHK bzw. nach Infarkt oder Bypassoperation sind ebenso wie herzgesunde Frauen häufiger von Depressionen betroffen als Männer [20, 23, 24]. Das Vorliegen einer Depression sagt bei bestehender KHK außerdem einen ungünstigen Krankheitsverlauf voraus [17, 21, $25,26]$. Patienten mit KHK, die außerdem unter Depressionen leiden, haben gegenüber Patienten, die nicht depressiv sind, ein etwa zwei- bis dreifach erhöhtes Risiko, im Verlauf ihrer Erkrankung ein kardiales Ereignis zu erleiden oder zu versterben [21, 22, 25, 26]. Es ist davon auszugehen, dass der ungünstige Einfluss der depressiven Symptomatik aufgrund von Verhaltenseffekten (z. B. durch Fehlernährung, körperliche Inaktivität) und physiologischen Effekten (z. B. autonome Dysbalance, Veränderungen in der Regulation der physiologischen Stressreaktion) vermittelt wird. $\mathrm{Ob}$ Patienten nach einem Herzinfarkt eine Depression entwickeln, ist dabei unabhängig von der Schwere des Herzinfarkts und einigen anderen medizinischen Faktoren [27]. Außerdem dauert die Symptomatik bei Frauen länger an [28]. Bjerkeset et al. [27] legen nahe, dass sich Frauen und Männer hinsichtlich der psychischen Belastung im Zeitverlauf unterscheiden. In den ersten beiden Jahren nach einem Herzinfarkt ist demnach das Risiko für Frauen, Depressionen oder Ängste zu entwickeln, im Vergleich zu Männern erhöht, während das Risiko für die Entwicklung depressiver Symptome für Männer erst 2 bis 5 Jahre nach dem Herzinfarkt erhöht war.

Pathologische Angst steht möglicherweise ebenfalls im Zusammenhang mit KHK-Inzidenz (für beide Geschlechter) [29] und ungünstiger Prognose nach Herzinfarkt [30].

Herzgesunde Personen mit feindseligen Eigenschaften (z. B. Zynismus, überdauernde negative Einstellungen gegenüber Anderen), haben eine leicht erhöhte Wahrscheinlichkeit, im Verlauf ihres Lebens eine KHK zu entwickeln. Dieser Effekt zeigt sich bei Männern deutlich ausgeprägter als bei Frauen [31]. Ebenso verschlechtert sich die Prognose von Patienten mit bestehender KHK, wenn sie sich als feindselig beschreiben [31]. Hier scheint es keine geschlechtsspezifischen Unterschiede zu geben [17].

Zum Zusammenhang zwischen dysfunktionalem Umgang mit Ärger (z. B. Unterdrücken von Ärger) und KHK gibt es noch keine gesicherte Datenlage. Bei Männern konnte bisher kein deutlicher Zusammenhang gefunden werden. Frauen dagegen, die in Konflikten mit ihren Ehepartnern ihren Ärger nicht zum Ausdruck brachten, hatten ein deutlich erhöhtes Risiko, an den Folgen einer KHK zu versterben $[6,32]$. 
Ein Jahr nach einem Herzinfarkt fanden Frasure-Smith et al. [24] keinen Zusammenhang zwischen unterdrücktem Ärger und dem kardialen Verlauf, während neuere Studien einen Zusammenhang zu einem erneuten kardialen Ereignis bei Frauen [33, 34] aufzeigten.

\section{Fazit}

In der Gesamtschau zeigt sich, dass in Bezug auf die psychosozialen Risikofaktoren mehr Gemeinsamkeiten als Unterschiede zwischen den Geschlechtern zu finden sind [17]. Dennoch sind diese ,feinen“ Unterschiede bedeutsam, da ihre Berücksichtigung bei der Erarbeitung psychosozialer Interventionen eine wichtige Rolle spielt.

\section{Genderspezifische Aspekte in der Wirksamkeit psychosozialer Interventionen}

Bei der noch übersichtlichen Anzahl experimentell untersuchter psychosozialer Interventionen wird zunehmend das Bestreben deutlich, geschlechtsspezifische Analysen der Wirksamkeit vorzunehmen.

Die ersten wissenschaftlichen Evaluationen schlossen selten Frauen in ihre Analysen ein und ermöglichten daher keine Aussagen zu genderspezifischen Unterschieden [35-37]. Diese Interventionen, die sich insbesondere auf die Verbesserung der Stressbewältigung konzentrierten, konnten das Wiederauftreten kardialer Ereignisse bei jüngeren Männern signifikant verbessern.

\section{Psychosoziale Interventionen im Einzelsetting}

Das Montreal Heart Attack Readjustment Trial (M-HART) [38] intervenierte ebenfalls durch die Unterstützung der Stressbewältigung. Die Intervention umfasste über 12 Monate hinweg monatliche telefonische Einzelkontakte und ggf. Hausbesuche durch Pflegepersonal. Die Intervention hatte bei Männern keinen Effekt auf die kardiale Sterblichkeit und insgesamt nur geringe Effekte im Hinblick auf Symptome von Depression und Angst. Bei Frauen der Interventionsgruppe schien sich die Sterblichkeit im Vergleich zur Kontrollgruppe sogar erhöht zu haben.

Neuere Studien wurden gezielt mit depressiven KHKPatientinnen und Patienten durchgeführt. Im Rahmen des Coronary Psychosocial Evaluation Studies Randomized Controlled (COPES) Trial [39] hatten Probanden mit kürzlich diagnostiziertem akutem Koronarsyndrom und persistierender depressiver Symptomatik in der Interventionsgruppe die Wahl, sich 6 Monate medikamentös oder psychotherapeutisch behandeln zu lassen. Pflegepersonal kümmerte sich regelmäßig darum, die Behandlung anzupassen. Geschlechtsspezifische Analysen zeigten, dass die
Intervention bei Männern und Frauen zu einer Verbesserung der depressiven Symptomatik führte, die lediglich bei den Frauen statistische Signifikanz erreichte. In der Interventionsgruppe ergab sich eine signifikante Reduktion schwerer kardialer Ereignisse. In Bypassing the Blues [40] wurden Patienten nach einer Bypassoperation bei depressiver Symptomatik telefonisch über 8 Monate hinweg von geschultem Pflegepersonal begleitet. Im Kontakt mit den Krankenschwestern konnten die Probanden unter mehreren Interventionsoptionen (Selbsthilferatgeber, antidepressive Medikation, Psychotherapie oder ,,watchful waiting“) wählen. Hier erzielten nur Männer durch die Intervention eine signifikante Verbesserung ihrer gesundheitsbezogenen Lebensqualität und eine Verringerung der depressiven Symptomatik im Vergleich zur Kontrollgruppe. Bei Frauen war der Effekt geringer ausgeprägt und nicht signifikant.

Das Canadian Cardiac Randomized Evaluation of Antidepressant and Psychotherapy Efficacy (CREATE) Trial [41] untersuchte eine zwölfwöchige Einzelintervention mit interpersoneller Kurzzeittherapie hinsichtlich ihrer Effekte bei depressiven KHK-Patienten. Im Vergleich zu kürzeren Gesprächen - ähnlich einer psychosomatischen Grundversorgung - konnte für beide Geschlechter kein Vorteil der spezifischen Psychotherapie nachgewiesen werden.

\section{Psychosoziale Interventionen im Gruppensetting}

Eine große Multicenterstudie in den USA (The Enhancing Recovery in Coronary Heart Disease (ENRICHD) Trial [42]) evaluierte eine Intervention an Patienten nach Infarkt, die komorbid eine depressive Episode bzw. Dysthymie aufwiesen oder über eine als gering wahrgenommene soziale Unterstützung berichteten. Die 6-monatige Intervention bestand in einer Kombination aus Einzel- und (gemischtgeschlechtlicher) Gruppenpsychotherapie mit kognitiv-verhaltenstherapeutischem Ansatz. Im Ergebnis konnte die Interventionsgruppe gegenüber der Kontrollgruppe nicht den beabsichtigten Überlebensvorteil erzielen [43]. Hinsichtlich der Besserung der Depressivität war die Interventions- der Kontrollgruppe in beiden Geschlechtern statistisch, jedoch nicht klinisch signifikant überlegen. Frauen der Interventionsgruppe wiesen gegenüber den Kontrollprobandinnen aber ein tendenziell erhöhtes Reinfarkt- bzw. Todesrisiko auf [43]. Nur weiße Männer profitierten prognostisch von der Intervention [44].

Cossette et al. [45] fanden in einer Sekundäranalyse der M-HART-Studie anhand der dokumentierten Interaktionen zwischen Patienten und Pflegekräften Hinweise darauf, dass Männer von Informationsvermittlung, Empfehlungen und Ansätzen kognitiver Umstrukturierung im Hinblick auf ihr Stresserleben profitierten. Frauen dagegen fühlten sich eher entlastet, wenn man ihnen aufmerksam zuhörte und sie ermutigte. 
Burell u. Granlund [46] berichteten, dass Männer in gemischtgeschlechtlichen Gruppen vornehmlich ihre Arbeitsbelastung schilderten, während Frauen eher Insuffizienzerleben, familiäre Belastungen und kritische Lebensereignisse thematisierten. Sie konzipierten daher ein geschlechtshomogenes Angebot, das sich auf die Anliegen der Frauen fokussiert.

Mit dem Stockholm Women's Intervention Trial for Coronary Heart Disease (SWITCHD) wurde eine psychosoziale Intervention für Frauen überprüft [47]. An der Intervention nahmen Frauen 4 Monate nach einer Revaskularisation unabhängig von ihrem psychischen Befinden teil. Die kognitiv-verhaltenstherapeutische Intervention fand im Rahmen von 20 Gruppensitzungen statt. Die Ergebnisse zeigen, dass die Intervention einen signifikant positiven Einfluss auf die Sterblichkeit hatte und somit auch Frauen mit ausgeprägter koronarer Herzerkrankung von einer psychosozialen Intervention profitieren können, wenn sie auf Frauen spezifisch ausgerichtet ist.

Das Secondary Prevention in Uppsala Primary Health Care Project (SUPRIM) überprüfte ebenfalls die Effekte einer auf kognitiver Verhaltenstherapie basierten Gruppenintervention hinsichtlich des Wiederauftretens kardialer Ereignisse bei KHK [48]. Die Intervention umfasste 20 zweistündige Sitzungen in geschlechtsgetrennten Gruppen. Das Interventionsprogramm hatte ohne Geschlechtsunterschied einen signifikant günstigen Einfluss auf das (Wieder-) Auftreten kardialer Ereignisse.

Basierend auf den Überlegungen, dass Frauen mit KHK von Interventionen, in denen sie unter sich sind, eher zu profitieren scheinen und telefonische Interventionen insgesamt hilfreich sein könnten, wenn die Gesprächspartnerinnen zuhören und ermutigen, wurde an der Abteilung für Psychosomatische Medizin und Psychotherapie der Universitätsmedizin Göttingen in Kooperation mit der KKH-Allianz eine telefonische Peer-support-Intervention (FrauenherzTelefon) entwickelt. Hierfür wurden Frauen mit koronarer Herzerkrankung speziell geschult, um ihre „Leidensgenossinnen", die eine depressive und/oder ängstliche Symptomatik angaben, durch aufmerksames Zuhören und das Berichten aus eigener Erfahrung zu unterstützen. Aktuell wird die Wirksamkeit dieser Intervention evaluiert.

Des Weiteren findet in Deutschland eine große multizentrische, experimentelle Untersuchung einer gestuften psychotherapeutischen Intervention für Patienten mit KHK (A Stepwise Psychotherapy Intervention for Reducing Risk in Coronary Artery Disease (SPIRR-CAD) [49]) statt. Patienten der Interventionsgruppe werden zunächst in drei Einzelsitzungen psychotherapeutisch behandelt. Wenn anschließend die depressiven Symptome fortbestehen, nehmen sie an einer 25 Sitzungen umfassenden Gruppenpsychotherapie teil, die auf kombinierten kognitiv-verhaltenstherapeutischen und psychodynamischen Konzepten beruht. Probanden der Kontrollgruppe erhalten neben der üblichen kardialen Behandlung ein psychosoziales Beratungsgespräch durch eine Studienschwester. Ziel der Intervention ist insbesondere eine Reduktion der depressiven Symptomatik nach 18 Monaten sowie u. a. die Verbesserung des kardialen Risikoprofils.

\section{Fazit}

Zusammenfassend zeigten Linden et al. [50] in einer Metaanalyse, dass psychosoziale Interventionen bei Herzpatienten die Sterblichkeit innerhalb der ersten beiden Jahre bei Männern, nicht aber bei Frauen signifikant reduzieren können. $\mathrm{Zu}$ einem ähnlichen Ergebnis kommen auch Low et al. [17] in ihrer Überblicksarbeit. Baumeister et al. [51] konnten dagegen in ihrer Metaanalyse bei depressiven KHK-Patienten nicht nachweisen, dass psychosoziale Interventionen die Sterblichkeit oder kardiale Ereignisse reduzieren. Die Ergebnisse der genannten neuen Studien flossen hier allerdings noch nicht mit ein. Es konnte jedoch ein klinisch bedeutsamer Effekt auf die depressive Symptomatik nachgewiesen werden.

Leider ist noch zu wenig darüber bekannt, wodurch psychosoziale Interventionen bei Männern und Frauen wirksam werden können. Die geschlechtsspezifischen Wirkfaktoren gilt es weiterhin zu untersuchen und gezielt in der Planung und Gestaltung von zukünftigen Interventionen zu berücksichtigen.

\section{Empfehlungen für die Praxis}

Die Beachtung der bekannten psychosozialen KHK-Risikofaktoren ist bei beiden Geschlechtern sinnvoll und wird von vielen Leitlinien empfohlen. Dabei sollte bei Frauen besonders auf Doppelbelastungen aus Beruf und Familie sowie Belastungen im häuslichen Umfeld geachtet werden. Depressionen, die bei beiden Geschlechtern mit der Entwicklung und Prognose der KHK assoziiert sind, kommen bei Frauen häufiger vor als bei Männern und erfordern eine systematische Beachtung und Behandlung. Hilfreich ist bei leichten Belastungen bzw. Störungen eine Versorgung im Rahmen der psychosomatischen Grundversorgung, ggf. ergänzt durch Selbsthilfeangebote (z. B. Selbsthilfegruppen oder das Frauenherz-Telefon). Bei deutlichen Ausprägungen kann an einen Facharzt für psychosomatische Medizin bzw. Psychiatrie oder an einen Psychotherapeuten verwiesen werden. In schweren Fällen stehen stationäre psychokardiologische Behandlungs- und Rehabilitationsangebote zur Verfügung. Eine differenzielle Therapieindikation lässt sich aus der derzeitigen Datenlage noch nicht ableiten. Eine Berücksichtigung geschlechtsspezifischer Themen 
und Interventionen wird jedoch empfohlen, auch wenn ihre Details weitere Forschung erfordern.

Acknowledgement This article is part of a supplement sponsored by Lilly Deutschland GmbH and Daiichi Sankyo Deutschland GmbH.

Interessenkonflikt Forschungsförderung zur Durchführung des Frauenherz-Projekts durch KKH-Allianz Interessenkonflikt Prof. Dr. Herrmann-Lingen: Vortragshonorar durch KKH-Allianz.

\section{Literatur}

1. Yusuf S, Hawken S, Ounpuu S (2004) Effect of potentially modifiable risk factors associated with myocardial infarction in 52 countries (the INTERHEART study): case-control study. Lancet 364(9438):937-952

2. Rozanski A, Blumenthal J, Davidson KW, Saab PG, Kubzansky L (2005) The epidemiology, pathophysiology, and management of psychosocial risk factors in cardiac practice: the emerging field of behavioral cardiology. J Am Coll Cardiol 55:637-651

3. Orth-Gomér K, Horsten M, Wamala SP et al (1998) Social relations and extent and severity of coronary artery disease. The Stockholm Female Coronary Risk Study. Eur Heart J 19:1648-1656

4. De Vogli R, Chandola T, Marmot MG (2007) Negative aspects of close relationships and heart disease. Arch Intern Med 167(18):1951-1957

5. Barefoot JC, Grønbæk M, Jensen G, Schnohr P, Prescott E (2005) Social network diversity and risks of ischemic heart disease and total mortality: findings from the Copenhagen City Heart Study. Am J Epidemiol 161(10):960-967

6. Eaker ED, Sullivan LM, Kelly-Hayes M et al (2007) Marital status, marital strain, and risk of coronary heart disease or total mortality: the Framingham Offspring Study. Psychosom Med 69:509-513

7. Orth-Gomér K, Wamala SP, Horsten M et al (2000) Marital stress worsens prognosis in women with coronary heart disease: the Stockholm Female Coronary Risk Study. JAMA 284(23):3008-3014

8. Karasek RA (1979) Job demands, job decision latitude, and mental strain: implications for job redesign. Admin Sci Quart 24(2):285-308

9. Orth-Gomér K, Leineweber C (2005) Multiple stressors and coronary disease in women. The Stockholm Female Coronary Risk Study. Biol Psychol 69:57-66

10. Karasek R, Baker D, Marxer F, Ahlbom A, Theorell T (1981) Job decision latitude, job demands, and cardiovascular disease: a prospective study of Swedish men. Am J Public Health 71(7):694-705

11. Siegrist J, Peter R, Junge A, Cremer P, Seidel D (1990) Low status control, high effort at work and ischemic heart disease: prospective evidence from blue-collar men. Soc Sci Med 31(10):1127-1134

12. Kuper H, Singh-Manoux A, Siegrist J, Marmot M (2002) When reciprocity fails: effort-reward imbalance in relation to coronary heart disease and health functioning within the Whitehall II study. Occup Environ Med 59(11):777-784

13. Kuper H (2003) Job strain, job demands, decision latitude, and risk of coronary heart disease within the Whitehall II study. J Epidemiol Community Health 57(2):147-153

14. Eaker ED (2004) Does job strain increase the risk for coronary heart disease or death in men and women? The Framingham Offspring Study. Am J Epidemiol 159(10):950-958
15. Slopen N, Glynn RJ, Buring JE, Lewis TT, Williams DR, Albert MA (2012) Job strain, job insecurity, and incident cardiovascular disease in the Women's Health Study: results from a 10-year prospective study. PLoS One 7(7):e40512

16. Rosengren A, Hawken S, Öunpuu S et al (2004) Association of psychosocial risk factors with risk of acute myocardial infarction in 11119 cases and 13648 controls from 52 countries (the INTERHEART study): case-control study. Lancet 364:953-962

17. Low CA, Thurston RC, Matthews KA (2010) Psychosocial factors in the development of heart disease in women: current research and future directions. Psychosom Med 72:842-854

18. Aboa-Éboulé C (2007) Job strain and risk of acute recurrent coronary heart disease events. JAMA 298(14):1652

19. Rugulies R (2002) Depression as a predictor for coronary heart disease. a review and meta-analysis. Am J Prev Med 23(1):51-61

20. Rudisch B, Nemeroff CB (2003) Epidemiology of comorbid coronary artery disease and depression. Biol Psychiatry 54(3):227-240

21. Lett HS, Blumenthal JA, Babyak MA, Sherwood A, Strauman T, Robins C, Newman MF (2004) Depression as a risk factor for coronary artery disease: evidence, mechanisms, and treatment. Psychosom Med 66(3):305-315

22. Nicholson A, Kuper H, Hemingway H (2006) Depression as an aetiologic and prognostic factor in coronary heart disease: a metaanalysis of 6362 events among 146538 participants in 54 observational studies. Eur Heart J 27:2763-2774

23. Lespérance F, Frasure-Smith N (2000) Depression in patients with cardiac disease: a practical review. J Psychosom Res 48:379-391

24. Frasure-Smith N, Lesperance F, Juneau M et al (1999) Gender, depression, and one-year prognosis after myocardial infarction. Psychosom Med 61:26-37

25. Barth J, Schumacher M, Herrmann-Lingen C (2004) Depression as a risk factor for mortality in patients with coronary heart disease: a meta-analysis. Psychosom Med 66:802-813

26. van Melle JP, de Jonge P, Spijkerman TA, Tijssen JGP, Ormel J, van Veldhuisen DJ, Van Den Brink RHS, Van Den Berg MP (2004) Prognostic association of depression following myocardial infarction with mortality and cardiovascular events: a metaanalysis. Psychosom Med 66:814-822

27. Bjerkeset O, Nordahl HM, Mykletun A, Holmen J, Dahl AA (2005) Anxiety and depression following myocardial infarction: gender differences in a 5-year prospective study. J Psychosom Res 58:153-161

28. Drory Y, Kravetz S, Hirschberger G (2003) Long-term mental health of women after a first acute myocardial infarction. Arch Physical Med Rehabil 84(10):1492-1498

29. Roest AM, Martens EJ, de Jonge P, Denollet J (2010) Anxiety and risk of incident coronary heart disease: a meta-analysis. J Am Coll Cardiol 56:38-46

30. Roest AM, Martens EJ, de Jonge P, Denollet J (2010) Prognostic association of anxiety post myocardial infarction with mortality and new cardiac events: a meta-analysis. Psychosom Med 72:563-569

31. Chida Y, Steptoe A (2009) The association of anger and hostility with future coronary heart disease. J Am Coll Cardiol 53(11):936-946

32. Harburg E (2003) Expressive/suppressive anger-coping responses, gender, and types of mortality: a 17-year follow-up (Tecumseh, Michigan, 1971-1988). Psychosom Med 65:588-597

33. László KD, Janszky I, Ahnve S (2010) Anger expression and prognosis after a coronary event in women. Int J Cardiol 140:60-65

34. Denollet J, Gidron Y, Vrints CJ, Conraads VM (2010) Anger, suppressed anger, and risk of adverse events in patients with coronary artery disease. Am J Cardiol 105(11):1555-1560 
35. Friedman M, Thoresen CE, Gill JJ et al (1986) Alteration of type A behavior and its effect on cardiac recurrences in post myocardial infarction patients: summary results of the recurrent coronary prevention project. Am Heart J 112(4):653-665

36. Burell G (1994) Modification of the type A behavior pattern in post-myocardial infarction patients: a route to cardiac rehabilitation. Int J Behav Med 1(1):32

37. Burell G (1996) Group psychotherapy in project new life: treatment of coronary prone behavior in coronary artery bypass graft surgery patients. In: Allan R, Scheidt S (Hrsg) Heart \& mind: the practice of cardiac psychology. American Psychological Association, Washington, DC, S 291-310

38. Frasure-Smith N, Lespérance F, Prince RH et al (1997) Randomised trial of home-based psychosocial nursing intervention for patients recovering from myocardial infarction. Lancet 350:473-79

39. Davidson KW, Rieckmann N et al(2010) Enhanced depression care for patients with acute coronary syndrome and persistent depressive symptoms: coronary psychosocial evaluation studies randomized controlled trial. Arch Intern Med 170(7):600-608

40. Rollman BL, Belnap BH (2011) The Bypassing the Blues trial: collaborative care for post-CABG depression and implications for future research. Cleve Clin J Med 78:4-12

41. Lespérance F, Frasure-Smith N, Koszycki D et al (2007) Effects of citalopram and interpersonal psychotherapy on depression in patients with coronary artery disease: the Canadian Cardiac Randomized Evaluation of Antidepressant and Psychotherapy Efficacy (CREATE) trial. JAMA 297(4):367-379

42. The ENRICHD Investigators (2000) Enhancing Recovery in Coronary Heart Disease Patients (ENRICHD): study design and methods. Am Heart J 139:1-9

43. The ENRICHD Investigators (2003) Effects of treating depression and low perceived social support on clinical events after a myocardial infarction: the Enhancing Recovery in Coronary Heart Disease Patients (ENRICHD) randomized trial. JAMA 289:3106-16
44. Schneiderman N, Saab PG, Catellier DJ et al (2004) Psychosocial treatment within sex by ethnicity subgroups in the Enhancing Recovery in Coronary Heart Disease Clinical Trial. Psychosom Med 66(4):475-483

45. Cossette S, Frasure-Smith N, Lespérance F (2002) Nursing approaches to reducing psychological distress in men and women recovering from myocardial infarction. Int J Nurs Stud 39:479-494

46. Burell G, Granlund B (2002) Women's hearts need special treatment. Int J Behav Med 9:228-242

47. Ort-Gomér K, Schneiderman N, Wang H-X et al (2009) Stress reduction prolongs life in women with coronary disease: The Stockholm Women's Intervention Trial for Coronary Heart Disease (SWITCHD). Circ Cardiovasc Qual Outcomes 2:25-32

48. Gulliksson M et al (2011) Randomized controlled trial of cognitive behavioral therapy vs standard treatment to prevent recurrent cardiovascular events in patients with coronary heart disease: Secondary Prevention in Uppsala Primary Health Care Project (SUPRIM). Arch Intern Med 171(2):134-40

49. Albus C, Beutel M, Deter H-C et al (2011) A stepwise psychotherapy intervention for reducing risk in coronary artery disease (SPIRR-CAD): rationale and design of a multicenter, randomized trial in depressed patients with CAD. J Psychosom Res 71:215-222

50. Linden W, Phillips MJ, Leclerc J (2007) Psychological treatment of cardiac patients: a meta-analysis. Eur Heart J 28:2972-2984

51. Baumeister H, Hutter N, Bengel J (2011) Psychological and pharmacological interventions for depression in patients with coronary artery disease. Cochrane Database Syst Rev 9:CD008012. 